\title{
Cystic Echinococcosis: Current Scenario and Future Prospective
}

\author{
J.K. Chamuah ${ }^{1}$, Amenti ${ }^{1}$, Siju, S. Jacob ${ }^{2}$, Lalchamliani $^{1}$ and D. Borkotoky ${ }^{1}$ \\ ${ }^{1}$ ICAR-NRC on Mithun, Medziphema, Nagaland-797106, India \\ ${ }^{2}$ ICAR - National Institute of Veterinary Epidemiology and Disease Informatics, \\ Yelahanka, Bangalore. India \\ *Corresponding author
}

\section{A B S T R A C T}

\section{Keywords}

Hydatid cyst,

Genotypes,

Molecular etc

Article Info

Accepted:

12 October 2019

Available Online:

10 November 2019
Cystic echinococcosis cause by Echinococcus granulosus is a zoonotic parasitic disease affecting domestic livestock with a wide host range including human being. Based on different molecular approaches, ten genotypes have been identified from different parts of the world. Apart from different livestock, Hydatid cyst is also cause of concern in mithun (Bos frontalis) from north eastern hill region of country. Robust surveillance data coupled with recombinant antigen will be a crucial step for developing diagnostic measure with candid antigen for immunoprophylaxis against this disease in near future.

\section{Introduction}

The North Eastern Region (NER) of the country comprising of 8 States namely Arunachal Pradesh, Assam, Manipur, Meghalaya, Mizoram, Nagaland, Tripura and Sikkim falls under the high rainfall zone and the climate ranges from subtropical to alpine. The geographical area is approximately 2,76,300 sq. $\mathrm{km}$, and the population density varies from 13 persons / sq. km in Arunachal Pradesh to 343 persons / sq. km. in Assam. About 55 per cent of total area is under forest cover and about 1.2 million hector is under cultivation while about 2.2 million ha is estimated to be not available for cultivation.
The livelihood of the peoples of NER is mainly based on agriculture, but the mountainous topography has brought limitations to extensive agricultural operations. Animal husbandry plays an important role in the economy in supplementing the income of the rural house hold by providing gainful employment to marginal and small farmers, particularly to the landless agricultural laborers. It also provides subsidiary occupation in semi-urban areas. The majority of the population in this region is traditionally dependent on livestock for their livelihood; and there are no taboos for consumption of meat. It is a common feature to observe that each and every rural family 
maintains a backyard livestock unit in NER. Parasitic diseases represent one of the major havoc to the livestock sector in the NER. Amongst these, cystic echinococcosis caused by the metacestode stage (hydatid cyst) of the smallest canine cestode, Echinococcus granulosus is an economically important disease in livestock. Economic losses arise not only from the condemnation of infected viscera, but also from reduction in yield and quality of meat, milk, wool, hide value, birth rate, and fecundity.

\section{International status}

Cystic echinococcosis (CE) is an important zoonotic disease affecting various species of livestock and humans, caused by metacestodes of dog tapeworms of the E. granulosus complex (Eg complex). CE represents an increasing public health and socio-economic concern in Egypt (Sadjjadi et al., 2006) and many areas of the world especially in many rural, grazing areas of Africa (Omer et al., 2010 \& Njoroge et al., 2002), Iran (Ahmadi, 2005), Romania (Bart et al., 2006), Asia (Torgerson et al., 2006) and Australia (Jenkins, 2006). Cystic echinococcosis (CE) has a worldwide geographical distribution. It is found in all continents, with the highest prevalence in parts of the Russian Federation and adjacent independent states, China, north and east Africa, Australia and South America. In Europe; it is present in every country or region. The annual incidence of human $\mathrm{CE}$ varies between $<1$ and $>8$ per 105 population with the exception of Ireland, Iceland and Denmark. It is most intensively endemic in parts of Spain, southern Italy, Sardinia, and in India. In a study from China a high seroprevalence of $25.5 \%$ was reported by the indirect hemagglutination assay (IHA) in a Tibetan population. The high prevalence reported in the above study may be because this study was conducted in nomadic population, who are at high risk of having an infection (Yu et al., 2008). Studies based on mitochondrial DNA analysis have demonstrated that E. granulosus is actually a complex of species/genotypes which exhibit a marked genetic variability. Therefore, at least ten distinct genotypes (G1-G10) have been identified within the E. granulosus complex (Cardona and Carmena, 2013). These include two sheep strains (G1 and G2), two bovid strains (G3 and G5), a horse strain (G4), a camel strain (G6), two pig strains (G7 and G9), and two cervid strains (G8 and G10). In addition, recent molecular evidence suggests that infections in wild carnivores are likely caused by a specific strain (G11) named $E$. felidis. This genotype has been documented in lions and hyenas (Huttner et al., 2008). Genotypes G1-G3 cluster firmly together to form the taxon, $E$. granulosus sensu stricto $(E$. granulosus s.s.). These variants have broad geographical distributions and a wide range of host specificity and are responsible (particularly G1) for most human infections. The more distantly related genotype cluster G6-G10 (E. canadensis) includes strains that are all infective to humans, but to a much lesser extent than those from E. granulosus s.s. (Cardona and Carmena, 2013). Among the ten genotypes of E. granulosus (EG) recognized worldwide, only 5 strains were known to infect humans including G1, G2, G5, G6, and G7 strains. The most frequent strain associated with human CE appears to be the sheep strain $(\mathrm{G} 1)$ and the highest rates of infection are recorded in communities involved in extensive sheep farming (Grosso et al., 2012).

\section{Indian scenario on hydatid cyst prevalence}

Hydatid disease is endemic in many parts of India and is a cause of serious concern due to increase in morbidity and considerable economic loss. In India several factors including cultural, educational, socioeconomical, agricultural and environmental 
contribute to the transmission of infection. In addition to this, lack of education and knowledge about the life cycle of the $E$. granulosus, as well as the lack of legislation for meat inspection and offal disposal at local abattoirs, contributes to domestic cycles of transmission. Studies on the sero-surveillance of cystic echinococcosis in India are scanty. Most of the prevalence studies are based on screening of slaughtered animals. For the control of disease it demands generation of an authentic sero-prevalence data which is lacking in India. Earlier studies from Western India demonstrated the presence of 4 different genotypes of the E. granulosus (genotype G1, G2, G3 and G5) in food producing animals (Gudewar et al., 2009) Pednekar et al., (2009), reported four genotypes of E. granulosus namely the sheep strain (G1), Tasmanian sheep strain (G2), Indian buffalo strain (G3) and cattle strain (G5) of E. granulosus in livestock of Maharashtra and adjoining areas in Western India. In Ludhiana, North India, buffalo strain (G3) and common sheep strain (G1) was found to infect the livestock. The predominant genotype was found to be G3 genotype $(63 \%)$ present in all species of livestock followed by the G5 $(19.56 \%)$, the G1 (13\%) and the G2 genotype (4.34\%). The G1 and G3 genotypes have been demonstrated to infect the livestock in North India (Singh et al., 2012).

Through the past five decades, significant phenotypic and genetic variabilities have been recognized and identified in various strains of E. granulosus isolated from different regions. Studies have revealed that the different strains of E. granulosus consist of heterogeneous groups of genetic variants, which may display variations in morphology, host specificity, development rate, pathogenicity and geographical distributions. Thus identification of strain types is very important in strategizing and implementing an Echinococcus control and management program.

\section{Epidemiological and economic significance}

Economic losses arise not only from the condemnation of infected viscera, but also from reduction in yield and quality of meat, milk, wool, hide value, birth rate, and fecundity by cystic echinococcosis. In addition to it, $\mathrm{CE}$ is considered an emerging and reemerging zoonotic disease in many parts of the world. Recently, the World Health Organization (WHO) has included echinococcosis as part of a Neglected Zoonosis subgroup in its 2008-2015 strategic plans for the control of neglected tropical diseases. Two hosts are involved in the life cycle of E. granulosus, the definitive host being carnivores and the intermediate hosts include ungulates both domestic and wild animals and humans. The adult tapeworm lives in the small intestine of carnivores and intermediate larval stages (hydatid cyst) develops in the internal organs of a wide range of mammalian species such as cattle, sheep, goats including humans which acquire the infection through accidental ingestion of the tapeworm eggs (Soulsby, 1986). Transmission and maintenance of echinococcosis is dependent on complex interactions of several factors including environmental, host and pathogen factors. A number of such factors are of local epidemiological significance and identification of such factors is of important in the effective implementation of control measures.

E. granulosus, formerly regarded as a single species with a high genotypic and phenotypic diversity, is now recognized as an assemblage of cryptic species, which differ considerably in morphology, development, host specificity (including infectivity/pathogenicity for humans) and other aspects. Genetic diversity exhibiting among the E. granulosus in different regions of the world is a growing concern among the research community. This diversity is reflected in the mitochondrial and 
nuclear genomes and has led to the construction of phylogenetic trees and hypotheses on the origin and geographic dispersal of various taxa. Based on phenotypic characters and gene sequences, E. granulosus (sensu lato) has by now been subdivided into E. granulosus sensu stricto (including the formerly identified genotypic variants G1-3), E. felidis (the former 'lion strain'), E. equinus (the 'horse strain', genotype G4), E. ortleppi (the 'cattle strain', genotype G5) and $E$. canadensis. The latter species shows the highest diversity and is composed of the 'camel strain', genotype G6, the 'pig strain', genotype G7, and two 'cervid strains', genotypes G8 and G10. The G1 genotype of E. granulosus s.s. (sheep strain) is globally the dominant species of Echinococcus responsible for the majority of zoonotic CE cases and this genotype itself may also infect other herbivore hosts (e.g. cattle, camels, donkeys and macropods). This extensive biologic variation in E. granulosus may influence lifecycle patterns, pathology, antigenicity, transmission dynamics, and sensitivity to chemotherapeutic agents. For example, E. canadensis G6-G7, E. equinus, E. granulosus sensu stricto and $E$. ortleppi are transmitted mainly through domestic cycles. The identification of Echinococcus species involved might therefore have implications for the design and development of prevention and control measures, diagnostic assays, and drugs.

\section{Initiative of ICAR-NRC on Mithun}

Parasitism is also a most common cause of reduce production in terms of meat and milk. The occurrences of different helminth parasite have been recorded in mithun (Bos frontalis). Their identity was also recorded both based on morphological followed by molecular identification from different parts of north eastern hilly region of India (Chamuah et al., 2009; Chamuah et al., 2016). Among parasites, Hydatid cyst was recorded most commonly among mithun population due to sharing of sylvatic cycle with wild carnivores like dog, fox etc (Chamuah et al., 2005). The pathological interactions of Hydatid cyst in different visceral organs have also recorded with 16\% incidence (Chamuah et al., 2017). Histopathological examination of hydatid cysts in the liver, showed extensive fibrous connective tissue proliferation with eosinophilic and lymphocytic infiltration, in lung, parenchyma showed severe emphysema with infiltration of alveolar macrophages and lymphocytes infiltration. The lung parenchyma showed severe emphysema with presence of the cyst contained collapse hydatid membrane. Surrounding the cyst, there was presence of alveolar macrophages with lymphocytic infiltration. Spleen showed cystic cavity formation with accumulation of few necrotic debris and scolices (Chamuah et al., 2017). A preliminary work has been done to identify the strain of E. granulosus present in Mithun in Arunachal Pradesh. Thirty-seven hydatid cysts were recovered over a period of 1 year from the slaughtered mithun in Arunachal Pradesh. The DNA was extracted from the germinal membrane of ten hydatid cysts and a partial sequence of $377 \mathrm{bp}$ of the nad1 gene was PCR amplified from these cysts. The PCR amplicons were purified sequenced and analyzed using BLAST. The phylogenetic analysis was done to compare these sequences with known strains/species of Echinococcus circulating globally. BLAST alignment of the 377-bp partial sequence of the nad 1 gene showed $100 \%$ identity with $E$. granulosus in 8 out of 10 Echinococcus isolates. The other two isolates showed $99 \%$ identity with E. ortleppi. The phylogenetic relationship of these ten Echinococcus isolates showed that eight $(80 \%)$ Echinococcus isolates from the mithun clustered with $E$. granulosus (genotypes G1-3) with strong bootstrap (1000) replicates. However, the other two isolates (20\%) clustered with $E$. ortleppi (genotype 5) (Chamuah et al., 2015). 


\section{Future perspective}

Although, the infection in domestic animals is usually asymptomatic and detected only at post-mortem inspection at the slaughter houses, yet it causes great economic loss through condemnation of infected organ, in particular liver. One of the major hurdles in understanding the pathogenicity of this parasite is the possession of extensive strain variation. Since different strains of Echinococcus show different biological and pathogenic behavior, identification of these strains and their incidence can help recognition of the biological cycle of the parasite.

Robust surveillance data is fundamental in order to show the burden of disease and to evaluate the progress and success of control programmes. However as for other neglected diseases, data is scarce and will need more attention if control programmes need to be implemented and measured. Surveillance for cystic echinococcosis in animals is difficult because the infection is asymptomatic in livestock.

Diagnosis and detection of Echinococcus granulosus (sensu lato) infection in animals is a prerequisite for epidemiological studies and surveillance of echinococcosis in endemic, reemergent or emergent transmission zones. It has been known for many years that sheep infected experimentally with $E$. granulosus can mount detectable specific IgG responses within weeks which serve as the basis for the development of serodiagnostic techniques. Serological tests based on crude parasitic extract/ hydatid cyst fluid results in cross reaction with other taenid species rendering it less sensitive. One of the important aspects for the control of disease is the availability of the current status of the disease in a particular geographical region. So there is an urgent need to develop a specific as well as sensitive serological assay for generating accurate serosurveillance data. It demands development of recombinant antigen based assays for diagnosis purpose.

\section{Conflict of interest}

There is no conflict of interest among the authors.

\section{References}

Ahmadi, N.A. (2005). Hydatidosis in camels (Camelus romedaries) and their potential role in the epidemiology of Echinococcus granulosus in Iran. Journal of Helminthology 79, 119-125.

Bart, J., Morariu, S., Knapp, J., Ilie, M., Pitulescu, M., Anghel, A., et al., Genetic typing of Echinococcus granulosus in Romania. Parasitol Res. 2006; 98: 130137.

Cardona, G.A. and Carmena, D. (2013). A review of the global prevalence, molecular epidemiology and economics of cystic echinococcosis in production animals. Veterinary parasitology 192, 10-32.

Chamuah, J. K. 2005. Studies on some aspects of parasites of mithun (Bos frontalis). M.V.Sc. Thesis submitted to Assam Agricultural University, Khanapara, and Guwahati-22.

Chamuah, J.K., Dutta, B., Borkotoky, D. (2017). Pathological studies on helminth parasitic infection in mithun (Bos frontalis). Journal of Parasitic Diseases DOI 10.1007/s12639-017-0913-7.

Chamuah, J.K., Perumal, P., Singh, V., Mech, A. and Borkotoky, D. (2013).Helminth parasites of mithun (Bos frontalis) -An overview. Indian Journal of Animal Sciences 83 (3): 235-237.

Chamuah, J.K., Raina, O.K., Lalrinkima, H., Jacob, S.S., Sankar, M., Sakhrie, A. (2016). Molecular characterization of veterinary important trematode and cestode species in the mithun (Bos 
frontalis) from north-east India. Journal of Helminthology 90(5): 577-582.

Chamuah. J.K., Raina, O.K., Lalrinkima, H., Jacob, S.S., Sankar, M., Sakhrie, A., Lama, S. and Banerjee, P.S. 2015. Molecular characterization of veterinary important trematode and cestode species in the mithun Bos frontalis from northeast India. Journal of Helminthology. Pp 1-6.

Grosso, G., Gruttadauria, S., Biondi, A., Marventano, S. and Mistretta, A. (2012). Worldwide epidemiology of liver hydatidosis including the Mediterranean area. World Journal of Gastroenterology 18(13), 1425-1437.

Gudewar, J., Pan, D., Bera, AK., Das, SK., Konar A., et al., (2009) Molecular characterization of Echinococcus granulosus of Indian animal isolates on the basis of nuclear and mitochondrial genotype. Mol Biol Rep 36: 1381-1385.

Huttner, M., Nakao, M., Wassermann, T., Siefert, L., Boomker, J.D., Dinkel, A., Sako, Y., Mackenstedt, U., Romig, T. and Ito, A. (2008). Genetic characterization and phylogenetic position of Echinococcus felidis (Cestoda: Taeniidae) from the African lion. International Journal for Parasitology 38, 861-868.

Jenkins, D.J. (2006). Echinococcus granulosus in Australia, widespread and doing well! Parasitology International 55 (Suppl), S203-S206.

Njoroge, E.M., Mbithi, P.M., Gathuma, J.M., Wachira, T.M., Gathura, P.B., Magambo, J.K. and Zeyhle, E. (2002). A study of cystic echinococcosis in slaughter animals in three selected areas of northern Turkana, Kenya. Veterinary parasitology 104, 85-91.

Omer, R., Dinkel, A., Romig, T., Mackenstedt, U., Elnahas, A., Aradaib, IE., et al., A molecular survey of cystic echinococcosis in Sudan. Vet Parasitol. 2010; 169: 340-346.

Pednekar, RP., Gatne, ML., Thompson, RC., Traub, RJ. (2009) Molecular and morphological characterisation of Echinococcus from food producing animals in India. Vet Parasitol 165: 5865.

Sadjjadi S. Present situation of echinococcosis in the Middle East and Arabic North Africa. Parasitol Int. 2006; 55: S197202.

Singh, BB., Sharma, JK., Tuli, A., Sharma, R., Bal, MS., Aulakh, RS., Singh, Gill, JP. (2012) Prevalence and morphological characterisation of Echinococcus granulosus from north India. J Parasit Dis: $1-5$.

Soulsby, E.J.L. (1986). Helminths, Arthropods and Protozoa of Domesticated Animals, seventh ed. The English Language Book Society. Bailliere and Tindall, London. pp.1- 809.

Torgerson, P.R., Oguljahan, B., Muminov, A.E., Karaeva, R.R., Kuttubaev, O.T., Aminjanov, M. and Shaikenov, B. (2006). Present situation of cystic echinococcosis in Central Asia. Parasitology International 55 (Suppl), S207-S212.

Yu, SH., Wang, H., Wu, XH., Ma, X., Liu, PY., Zhao, YM., et al., (2008) Cystic and alveolar echinococcosis: an epidemiological survey in a Tibetan population in southeast Qinghai, China. Jpn J Infect Dis 61:242-6.

\section{How to cite this article:}

Chamuah, J.K., Amenti, Siju, S. Jacob, Lalchamliani and Borkotoky, D. 2019. Cystic Echinococcosis: Current Scenario and Future Prospective. Int.J.Curr.Microbiol.App.Sci. 8(11): 1546-1551. doi: https://doi.org/10.20546/ijcmas.2019.811.179 